\title{
Metaheurísticos para Solución del Problema de Ruteo en la Recuperación de Medicamentos Sobrantes y Suministros en Hospitales
}

\author{
Rodrigo A. Gómez ${ }^{1}$, Fernando Salazar ${ }^{2}$ y Nicolás Rincón ${ }^{3}$ \\ (1) Escuela Superior en Administración de Cadena de Suministro, Politécnico Colombiano Jaime Isaza \\ Cadavid, Carrera 48 \#7-151, Medellín, Colombia. (e-mail: rodrigomezm1986@gmail.com) \\ (2) Facultad de Ciencias Económicas y Administrativas, Pontificia Universidad Javeriana, Carrera 7ª \#40-62, \\ Bogotá, Colombia. (e-mail: salazar.fernando@javeriana.edu.co) \\ (3) Facultad de Ingeniería, Pontificia Universidad Javeriana, Carrera 7ª \#40-62, Bogotá, Colombia \\ (e-mail: nicolas.rincon@javeriana.edu.co)
}

Recibido Jun. 27, 2018; Aceptado Sep. 6, 2018; Versión final Oct. 11, 2018, Publicado Abr. 2019

\begin{abstract}
Resumen
Este artículo tiene como objetivo formular y solucionar un problema de ruteo para la recogida de medicamentos sobrantes y suministros en el mínimo tiempo posible. Para resolver el problema, dos metaheurísticos denominados recocido simulado (RS) y enjambre de partículas (PSO) son aplicados. Adicionalmente, factores como tamaño de lista de recolección (TLR), tamaño de flota de carros de recolección (TFCR), así como cantidades de habitaciones en el hospital son modelados. De la validación experimental se detectó que los niveles del metaheurístico PSO con el nivel TLR de 250 productos, genera menores tiempos de recogida con valores de 58,30 y 57,30 minutos / conjunto de rutas; en tanto, una combinación de los niveles TLR de 400 productos, con el metaheurístico RS, produce el menor tiempo promedio de ruteo con valores de 99,33 y 101,24 minutos /conjunto de rutas. Estos resultados mostraron la efectividad de los metaheurísticos para solucionar el problema ruteo en logística hospitalaria.
\end{abstract}

Palabras clave: ruteo; logística hospitalaria; enfriamiento simulado; enjambre de partículas

\section{Metaheuristics for Solving the Routing Problem of Collecting Leftover Medicines and Supplies in Hospitals}

\begin{abstract}
This article aims to formulate and solve a routing problem for the collection of surplus leftover medicines and supplies in the minimum possible time. To solve the problem, two metaheuristics called simulated annealing (RS) and particle swarm optimization (PSO) are applied. Additionally, factors such as collection list size (TLR), size of collection car fleet (TFCR), as well as hospital room quantities are modeled. From the experimental validation it was detected that the levels of the PSO metaheuristic with the TLR level of 250 products, generates shorter collection times with values of 58.30 and 57.30 minutes / set of routes. Meanwhile, a combination of the TLR levels of 400 products, with the RS metaheuristic, produces the lowest average routing time with values of 99.33 and 101.24 minutes / set of routes. These results showed the effectiveness of metaheuristics to solve the problem of routing in hospital logistics.
\end{abstract}

Keywords: routing; hospital logistics; simulated annealing; particle swarm 


\section{INTRODUCCIÓN}

La gestión logística en los hospitales cubre desde el aprovisionamiento, la prestación de los servicios, gestión de inventario, distribución de medicamentos y equipos, así como logística inversa (Kriegel et al., 2013). La logística hospitalaria impacta en la productividad y la satisfacción de los requerimientos de los usuarios (Park et al., 2014). La logística inversa en los hospitales presenta diferentes alcances según las características de su sistema logístico por lo cual puede ser dividido en dos enfoques. Un primer enfoque consiste en la gestión y recolección de sobrantes de medicamentos, dispositivos médicos entre otros suministros que pueden ser recuperados para prestar otros servicios. Un segundo enfoque es la gestión externa de residuos hospitalarios a los cuales se les implementan procesos logísticos tales como clasificación, recolección y transporte para garantizar una adecuada disposición final según la normatividad de los países.

Una revisión al estado del arte es realizada para detectar los enfoques publicados en la literatura científica acerca de la logística hospitalaria. A partir de una revisión del estado arte se identifica que los artículos en logística hospitalaria se dividen principalmente en dos grupos. El primer grupo artículos relacionados con la gestión de inventario y distribución. El segundo grupo se consideran artículos que formulan y solucionan problemas de ruteo en servicios hospitalarios. En el primer grupo Al-Qatawneh y Hafeez (2011) modelan una clasificación de inventarios multicriterio para logística hospitalaria buscando optimizar los costos logísticos y garantizar los niveles de servicio planeados. Bhakoo et al., (2012) desarrollan un modelo colaborativo de gestión de inventarios en la cadena de suministro que considera prácticas y factores claves para contribuir a su disponibilidad en la prestación de servicios en la logística hospitalaria. Battini et al., (2013) desarrolla un modelo que integra la gestión de inventario de medicamentos y redes de distribución con el fin de obtener los menores costos de servicio y diseñar rutas de transporte de mínimo tiempo.

Debe resaltarse que ninguno de los artículos revisados formula o soluciona problemas de ruteo de mínimo tiempo o costo para distribuir o recuperar productos en las actividades de logística hospitalaria. En el segundo grupo de artículos, Jlassi et al., (2012) formulan un problema denominado dial ride and emergency para servicios de ambulancia que se resuelve con un modelo de programación entera mixta. Kergosien et al., (2013) desarrollan dos metaheurísticos denominados búsqueda Tabú y Algoritmo genético que permite solucionar el problema de ruteo de colaboradores edificios dentro de un hospital los cuales transportan suministros y prestan servicios a los pacientes. Supakdee et al., (2013) implementan un algoritmo denominado Agrupación de localización por enrutamiento (Clustering-Locating-Routing) para solucionar un problema de ruteo y asignación de ubicación de instalaciones hospitalarias para suministrar medicamentos y productos médicos de una manera efectiva.

Ganesh, Narendran y Anbuudayasankar (2014) formulan un problema de ruteo de vehículos que genere costos efectivos logísticos para un banco de sangre el cual se soluciona utilizando un algoritmo genético (Genetic Algorithm - GA) y enfriamiento simulado (Simulated Annealing -SA). A partir de los resultados de la revisión de los antecedentes, no se detectaron artículos que aborden el problema de ruteo de mínimo tiempo dentro de los hospitales para la recogida o recuperación de medicamentos sobrantes, equipos móviles o suministros que puedan ser reutilizados entre otros servicios. Adicionalmente, no se identificaron artículos que modelaran el problema de ruteo para la disposición final de residuos en la logística de la cadena de suministro hospitalaria, sino que se enfoca en problemas de ruteo de ambulancias o distribución de suministros y otros estudios y avances que han aportado a la temática desde el enrutamiento de vehículos, considera aspectos como el topográfico y emisiones (Suarez et al., 2018).

El presente artículo tiene como objetivo formular un problema de ruteo para la recogida de medicamentos sobrantes y suministros desde las habitaciones o centro de servicios médicos dentro de un hospital hasta el almacén en el mínimo tiempo posible considerando el uso de una flota de equipos homogéneos (igual capacidad y velocidad de desplazamiento) así como la contaminación cruzada de los productos a manipular. Para solucionar el problema son implementados un metaheurístico de búsqueda tabú (Tabú Search - TS) y un metaheurístico de enfriamiento simulado. Para alcanzar el objetivo propuesto el artículo se estructura en las siguientes secciones. En la segunda sección, se describe el problema de ruteo de mínimo tiempo posible para la recogida de medicamentos sobrantes y suministros dentro de hospitales que incluye la representación del hospital, los equipos de transporte interno utilizado entre otros. En la tercera sección, se representa el modelo algebraico del problema de ruteo bajo modelamiento. En la cuarta sección, se modela el metaheurístico de búsqueda tabú (TS) y enfriamiento simulado (SA) que permiten solucionar el problema de ruteo. En la quinta sección, se realiza el análisis estadístico y la discusión de los resultados del problema de ruteo en estudio. Finalmente, en la sexta sección se presentan las conclusiones y trabajos futuros que se derivan del presente artículo. 


\section{MÉTODO}

En el análisis y aplicación de múltiples soluciones a problemáticas de carácter logístico y de optimización, se han estudiado heurísticas y meta heurísticas como: Algoritmos genéticos, Simulated Annealing, Tabú Search, entre otros; y se han desarrollado diferentes meta-heurísticas que modifican o perfeccionan las heurísticas a través de combinaciones de ellas o inclusión de procesos dentro de los algoritmos originales mejorando el tiempo de obtención de la solución, ampliando el espacio de soluciones o, dirigiendo las soluciones hacia un espacio del universo preestablecido o deseable (Talbi, 2009; y Rodríguez et al., 2017).

\section{Descripción del problema}

El problema de ruteo de mínimo tiempo posible para la recogida medicamentos y suministros sobrantes se representa para un hospital de mínimo 150 habitaciones que incluye locaciones para cirugías o atención de servicios hospitalarios. Se considera que el hospital tiene un almacén central desde el cual se inicia y finaliza las rutas de recogida de los medicamentos sobrantes y los suministros reutilizables. Se seleccionan estos productos para su recolección debido a su representación en los costos operacionales de los hospitales (alrededor de $70 \%$ ) y su posibilidad de volver a asignar a otros pacientes cuando no han sido utilizados. Por esta razón, se genera una oportunidad de eficiencia en los costos y la logística del hospital.

Para la recolección se utiliza una flota de vehículos de recogida manuales que tienen una capacidad de carga $\mathrm{C}$ que son manipulados colaboradores. Los vehículos de recogida tienen divisiones que permiten separar los medicamentos y suministro, así como evitar contaminación cruzada entre los productos. La operación de recogida consiste en ejecutar una ruta que inicia en el almacén central y visita cada una de las habitaciones o centros de servicios dentro del hospital para recoger la cantidad (QI) de medicamentos sobrantes o suministros reportados en el sistema de información hospitalario, así como su localización física dentro del hospital. El sistema de información hospitalario integra módulos de aprovisionamiento, prestación del servicio, gestión de inventarios y almacenes, finanzas, gestión humana, mantenimiento y gestión logística. En el módulo de gestión de prestación del servicio se registra como inventario sobrante los medicamentos y suministros que se programa su uso pero que no se utilizan. Esta información de inventarios es la entrada para solucionar el problema de ruteo de recogida, de acuerdo a la estructura mostrada en la figura 1:

\begin{tabular}{|c|c|c|c|c|c|c|c|c|c|}
\hline \multirow{3}{*}{ Piso 1} & \multirow{3}{*}{$\begin{array}{c}\text { Almacén de } \\
\text { medicamientos } \\
\text { y suministros }\end{array}$} & \multirow{3}{*}{ Ascensor } & Habitación 1 & Habitación 2 & Habitación 3 & Habitación 4 & Habitación 5 & Habitación 6 & Habitación 7 \\
\hline & & & \multicolumn{7}{|c|}{ Pasillo } \\
\hline & & & Habitación 1 & Habitación 2 & Habitación 3 & Habitación 4 & Habitación 5 & Habitación 6 & Habitación 8 \\
\hline & \multirow{3}{*}{ Piso 2} & \multirow{3}{*}{ Ascensor } & Habitación 9 & Habitación 10 & Habitación 11 & Habitación 12 & Habitación 13 & Habitación 14 & Habitación 15 \\
\hline & & & \multicolumn{7}{|c|}{ Pasillo } \\
\hline & & & Habitación 16 & Habitación 17 & Habitación 18 & Habitación 19 & Habitación 20 & Habitación 21 & Habitación 22 \\
\hline & \multirow{3}{*}{ Piso 3} & \multirow{3}{*}{ Ascensor } & Habitación 23 & Habitación 24 & Habitación 25 & Habitación 26 & Habitación 27 & Habitación 28 & Habitación 29 \\
\hline & & & \multicolumn{7}{|c|}{ Pasillo } \\
\hline & & & Habitación 30 & Habitación 31 & Habitación 32 & Habitación 33 & Habitación 34 & Habitación 35 & Habitación 36 \\
\hline & \multirow{3}{*}{ Piso $m$} & \multirow{3}{*}{ Ascensor } & Habitación 37 & Habitación 38 & Habitación 39 & Habitación 40 & Habitación 41 & Habitación 42 & Habitación 43 \\
\hline & & & \multicolumn{7}{|c|}{ Pasillo } \\
\hline & & & Habitación 44 & Habitación 45 & Habitación 46 & Habitación 47 & Habitación 48 & Habitación $j$ & Habitación $h$ \\
\hline
\end{tabular}

Fig. 1: Estructura de las habitaciones y pasillos en el hospital para el ruteo de recogida

Las rutas de recogida inician en el almacén de medicamentos y suministros, visitan habitaciones $(h, j)$ en los diferentes pisos. Además, las rutas están restringidas por la capacidad de carga de los vehículos, así como la condición de contaminación cruzada que exista entre los productos a recoger. De otra parte, una habitación o centro de servicio es visitada una sola vez en una ruta de recogida debido a la eficiencia operacional del ruteo logística buscando eficiencia en el tiempo y uso de los recursos. Al finalizar la ruta el vehículo regresa al almacén central donde los medicamentos y suministros se ingresan al inventario. El problema de ruteo de recogida considera matrices de origen y destino que contienen las distancias, así como los tiempos entre el almacén central y las diferentes habitaciones y los centros de servicios dentro del hospital. Adicionalmente, se considera que la velocidad de los vehículos de recogida es constante y la congestión en los pasillos es pasillos no es significativa. 


\section{Formulación del problema}

A continuación, se representa la formulación algebraica del problema de ruteo para la recogida de medicamentos sobrantes y suministros en el menor tiempo posible. La formulación algebraica que se presenta se compone de conjuntos e índices, parámetros, función objetivo, variables de decisión y restricciones, mostradas en las tablas 1 a 3 :

Tabla 1: Conjuntos e índices

\begin{tabular}{ll}
\hline$r \in R$ & $\begin{array}{l}\text { Ruta } \mathrm{r} \text { asociado a un conjunto de rutas } R \text { para recoger los medicamentos sobrantes y } \\
\text { suministros. }\end{array}$ \\
$j, h \in E$ & Habitaciones en el hospital \\
$g \in E$ & Subconjunto de habitaciones en el hospital \\
$k \in K$ & Carros recolectores disponibles en el hospital. \\
$i \in I$ & $\begin{array}{l}\text { Medicamento o suministro } i \text { asociado a la lista de productos a recuperar desde las } \\
\text { habitaciones hasta el almacén central del hospital }\end{array}$ \\
$C S R_{R}$ & Conjunto de solución de rutas $r \in R$ \\
\hline
\end{tabular}

Tabla 2: Parámetros del modelo

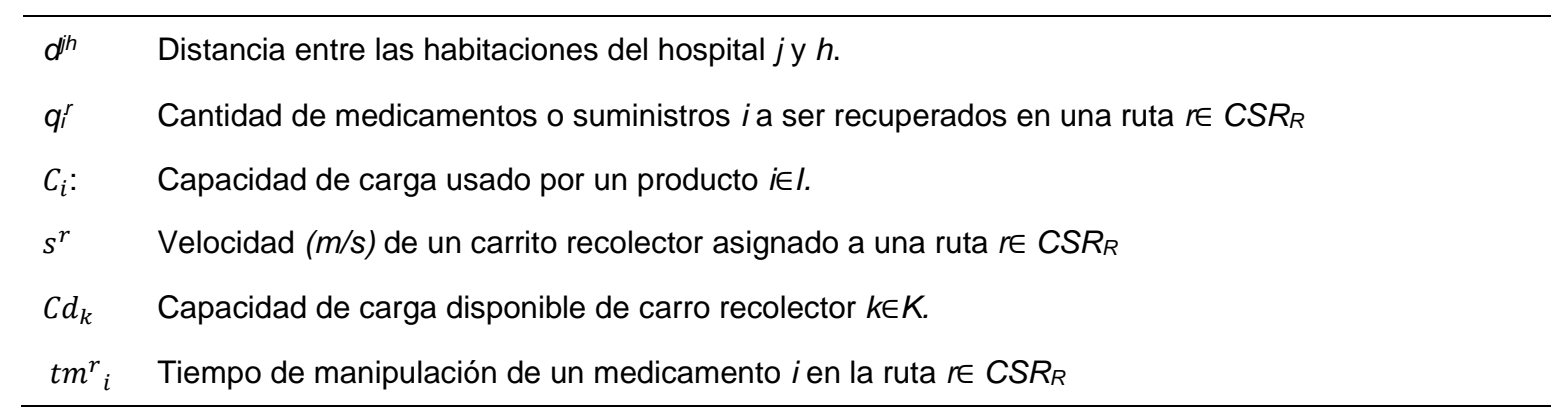

Tabla 3: Variables de decisión

\begin{tabular}{|c|c|}
\hline$X^{r}{ }_{j h}=$ & $\begin{array}{l}\text { 1, Si una habitación jes visitada inmediatamente después de una habitación } h \text { en una } \\
\text { ruta de recolección } r \in C S R_{R}\end{array}$ \\
\hline & 0, De otro modo \\
\hline$Z_{i}^{r}=$ & $\begin{array}{l}\text { 1, Si un medicamento o suministro } i \text { es asignado a una ruta } r \in C S R_{R} \\
0 \text {, De otro modo }\end{array}$ \\
\hline$Y_{k}^{r}=$ & $\begin{array}{l}\text { 1, Si un carro recolector } k \text { es programado a una ruta } r \in C S R_{R} \\
0 \text {, De otro modo }\end{array}$ \\
\hline$M_{h}^{r}=$ & $\begin{array}{l}\text { 1, Si una habitación } h \text { es visitada dentro de una ruta } r \in C S R_{R} \\
0, \text { De otro modo }\end{array}$ \\
\hline
\end{tabular}

$\operatorname{Min} T=\sum_{r \in R} \sum_{k \in K} \sum_{j \neq h \in E} \frac{d^{r}{ }_{j h}}{V^{r}} \cdot Y_{k}^{r}+\sum_{r \in R} \sum_{i \in I} q_{i}^{r} \cdot t m^{r}{ }_{i} \cdot Z^{r}$

Sujeto a:

$\sum_{r \in R} Z_{i}^{r}=1 \forall i \in I$

$\sum_{i \in I} q_{i}^{r} C_{i} Z_{i}^{r} \leq C d_{k} Y_{k}^{r} \forall r \in R, k \in K$

$\sum_{j \in g, j \in E \backslash g} X^{r}{ }_{j h} \geq M^{r}{ }_{h} \forall r \in R, h \in E$ 


$$
\begin{aligned}
& \sum_{h \in E, h \neq j} X_{j h}^{r}=M^{r}{ }_{j} \forall r \in R, j \in E \\
& \sum_{j \in E, j \neq h} X^{r}{ }_{j h}=M^{r}{ }_{h} \forall r \in R, h \in E \\
& Z^{r}{ }_{i}, Y_{k}{ }^{r}, M_{h}{ }^{r} \in\{0,1\}
\end{aligned}
$$

La función objetivo (1) minimiza el tiempo total para la recogida de medicamentos y suministros sobrantes de las habitaciones de un hospital. La restricción (2) controla que se asigna cada medicamento o suministro $i$ a una $r \in C S R_{R}$. La restricción (3) controla que la capacidad de un carrito recolector $k \in K$ no es sobrepasada por la cantidad de medicamentos sobrantes o suministros i programados a una ruta $r \in C_{S} R_{R}$. La restricción (4) evita subrutas en el conjunto de $g$ habitaciones en el hospital en estudio. Las restricciones (5) y (6) aseguran que cada habitación del hospital tiene un predecesor y un sucesor en una ruta $r \in C_{S} R_{R}$. Por último, la restricción (7) establece que las variables de decisión del problema sean positivas.

\section{Metaheurísticos de Recocido Simulado (RS) y Optimización de Enjambre de Partículas (OEP) para la solución del problema}

En esta sección se desarrollan dos metaheurísticos denominados Enfriamiento Simulado y Optimización de Enjambre de Partículas (OEP) o (Particle Swarm Optimization (PSO)) que permiten resolver el problema de ruteo de recogida de medicamentos sobrantes y suministros en el menor tiempo posible para hospitales. Para cada metaheurístico se elabora un diagrama de flujo que contiene sus parámetros, así como la secuencia lógica de etapas para solucionar el problema en estudio. Debe resaltarse que el metaheurístico de enjambre de partículas (OEP) se le realiza una adaptación ya que el problema de ruteo en formulación tiene soluciones discretas. Debe resaltarse que los metaheurísticos que se desarrollan para abordar este problema no habían sido detectados en la literatura de allí su novedad.

En el modelamiento de los metaheurísticos OEP y RS, se representa una matriz de distancias entre las habitaciones y el punto de inicio del ruteo logístico para la recogida de los medicamentos e insumos restantes. Adicionalmente, se representa una flota de equipos de manejo de materiales con capacidad y velocidad que son parametrizables. Por lo tanto, los metaheurísticos son adaptables a las características del problema representado. Los diagramas de flujo para los metaheurísticos OEP y RS, se presentan en la figura 2 y RS de la figura 3 , respectivamente.

Tabla 4: Definición de variables

$\begin{array}{cl}v^{t+1}{ }_{i j} & \text { Velocidad de una partícula } i^{r} \text { en una dimensión } j \text { en el tiempo } t+1 \\ v^{t}{ }_{i j} & \text { Velocidad de una partícula } i^{r} \text { en una dimensión } j \text { en el tiempo } t \\ \omega & \text { Factor de inercia } \\ c_{1} & \text { Constante positiva que representa un componente cognitivo para el metaheurístico PSO } \\ r^{t}{ }_{1 j} & \text { Número aleatorio entre [0,1] } \\ P^{t}{ }_{\text {best,i }} & \text { Mejor posición de la partícula } i^{r} \text { en la dimensión } j \text { en el tiempo } t \\ X^{t}{ }_{i j} & \text { Posición de la partícula } i^{r} \text { en la dimensión j en el tiempo } t \\ c_{2} & \text { Constante positiva que representa un componente social para el metaheurístico PSO } \\ r^{t}{ }_{2 j} & \text { Número aleatorio entre [0,1] } \\ G_{b e s t} & \text { Mejor posición de la partícula } i^{r} \text { en la dimensión } j \text { calculada hasta ahora }\end{array}$




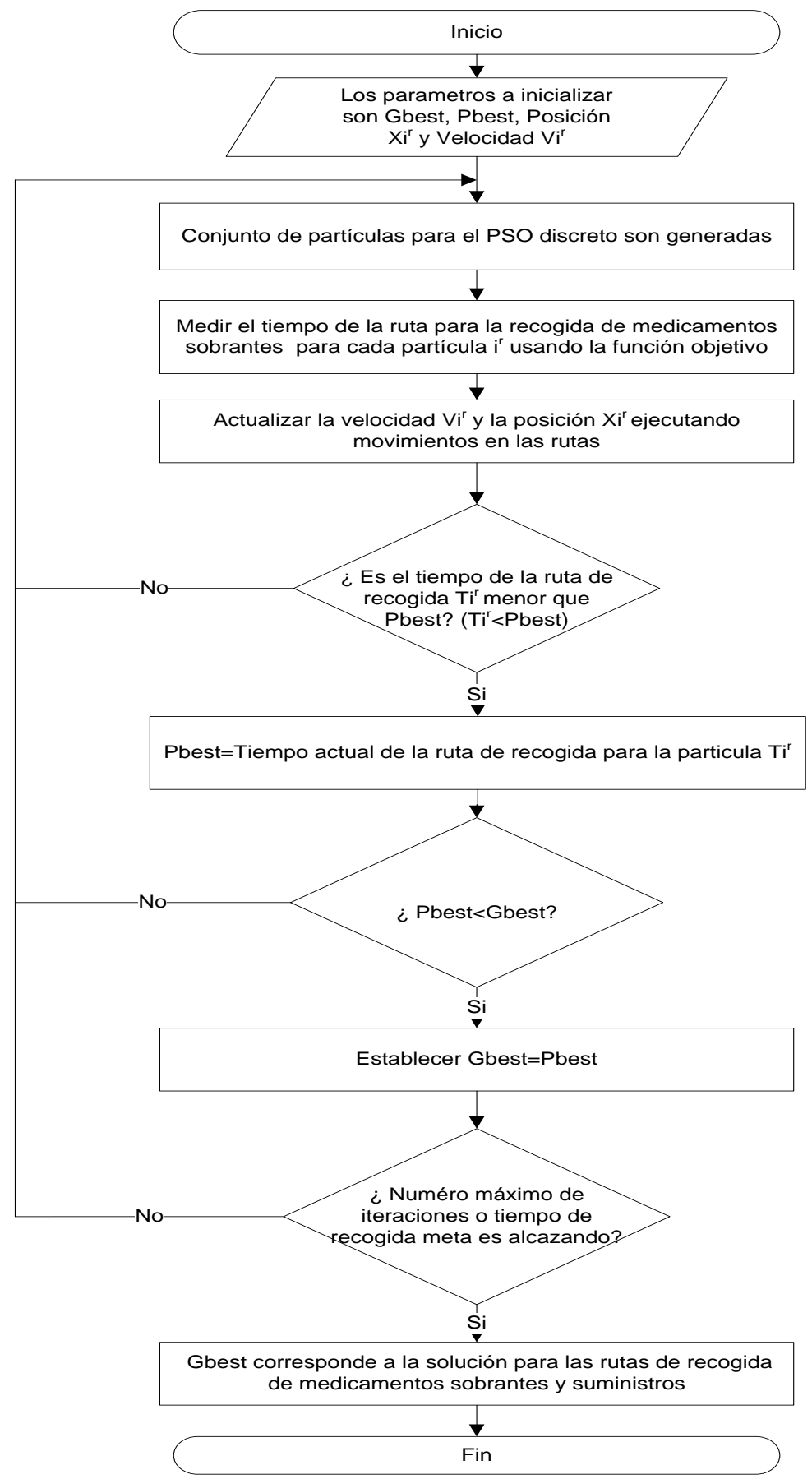

Fig. 2: Diagrama de metaheurístico PSO discreto para solucionar problema de ruteo

El metaheurístico OEP discreto comienza con la inicialización de los parámetros de posición $X{ }^{r}{ }^{r}$ y velocidad $V i{ }^{r}$ así como Gbest (mejor solución global o mejor conjunto de rutas) y Pbest (Mejor solución para conjunto de partículas) que permiten representar las mejores soluciones de rutas para el problema en logística hospitalaria. Posteriormente un conjunto inicial de partículas, son generadas aleatoriamente. A este conjunto inicial de partículas que representa las rutas de recogida se les mide el tiempo inicial utilizando la función objetivo definido para el problema (Eq. 1). Luego se actualiza la posición $X^{r}{ }^{r}$ y velocidad $V i^{r}$ realizando movimientos que permiten generar el nuevo conjunto de rutas de recogida de medicamentos sobrantes y suministro de los servicios en la logística hospitalaria empleando las siguientes ecuaciones. La velocidad $\mathrm{Vir}^{\mathrm{r}}$ es calculada utilizando la Eq. (8).

$$
v^{t+1}{ }_{i j}=\omega v^{t}{ }_{i j}+c_{1} r^{t}{ }_{1 j}\left[P^{t}{ }_{\text {best }, i}-X^{t}{ }_{i j}\right]+c_{2} r^{t}{ }_{2 j}\left[G_{\text {best }}-X^{t}{ }_{i j}\right]
$$

Una nueva posición para la partícula es calculada utilizando la Eq. (9):

$$
x_{i^{r+1}}=x_{i^{r t+}} v^{t+1}{ }_{i j}
$$


Posteriormente, se evalúa si los tiempos de la ruta de recogida Tir menor que Pbest. En caso de que esta condición se cumpla, se actualiza el tiempo de Tícomo el nuevo Pbest en el metaheurístico PSO de lo contrario se regresa a la etapa de generar un conjunto de partículas para el OEP discreto. Seguidamente, se evalúa si el Pbest calculado es menor que Gbest (mejor solución de rutas de recogida generadas). En el escenario que esta condición se cumpla se establece el Pbest como el nuevo Gbest para el metaheurístico PSO de lo contrario se regresa a la etapa de generar un conjunto de partículas para el OEP discreto. Posteriormente, se determina si el número máximo de iteraciones o el tiempo de ruteo de recogida meta ha sido alcanzado. En caso de que esta condición se cumpla se establece que el Gbest actual es la mejor solución para el problema de rutas de recogida de medicamentos sobrantes y suministros de mínimo tiempo posible calculado para el problema en solución de lo contrario se regresa a la etapa de generar un conjunto de partículas para el OEP discreto.

El segundo metaheurístico denominado RS (Recocido Simulado) que se presenta en la figura 3, se representa el diagrama de flujo con las etapas para solucionar el problema de recogida de medicamentos e insumos.

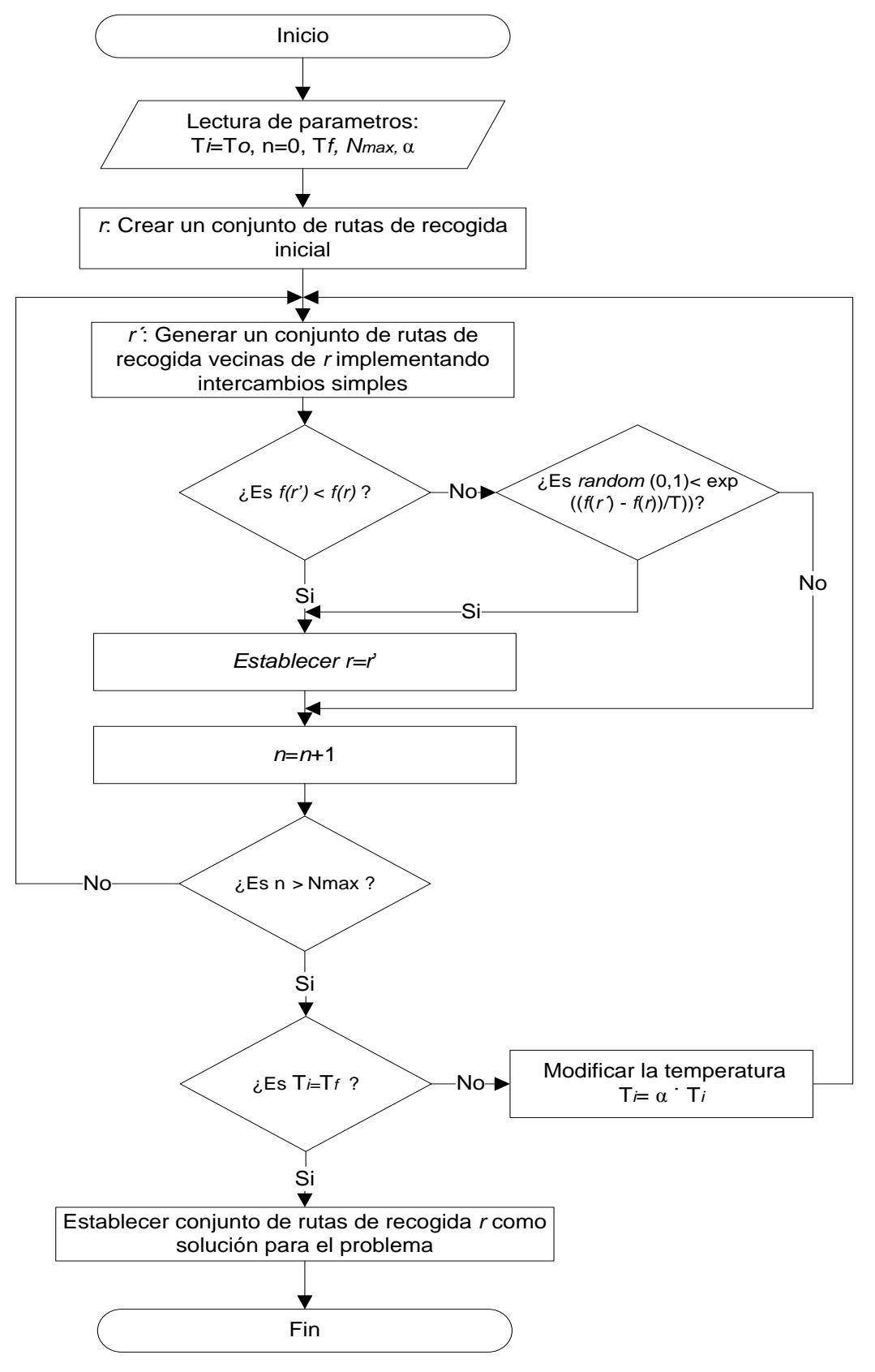

Fig. 3: Diagrama enfriamiento simulado para solucionar problema de ruteo

El metaheurístico de enfriamiento simulado comienza con la lectura de $\mathrm{T} i=\mathrm{To}$ (temperatura inicial), $\mathrm{n}=0$ (primera iteración), Tf (temperatura final), $N \max$ (interacción máxima por nivel de temperatura) y a (factor de enfriamiento). La primera etapa consiste en generar un conjunto de rutas iniciales las cuales se denominan $r$ a las cuales se les mide el tiempo de ruteo para la recogida de los medicamentos sobrantes y los suministro. 
En una segunda etapa se crea un conjunto de rutas $r^{\prime}$ a partir de la implementación de movimientos de intercambio o inserción sobre el conjunto de rutas inicial o actual $r$. Al conjunto de rutas $r$ ' se le mide el tiempo utilizando la función objetivo definida en la Eq.(1). En la cuarta etapa, se evalúa si el tiempo obtenido para el conjunto de ruta generado con la implementación de movimientos $r^{\prime}$ es menor al conjunto de rutas $r\left(\left(f\left(r^{\prime}\right)<f(r)\right)\right.$. En caso de que esta condición se cumpla, el conjunto de rutas $r^{\prime}$ se establece como el conjunto de rutas actual r para el metaheurístico de enfriamiento simulado. De lo contrario, la condición se evalúa la Eq. (10):

$$
\text { Random }(0,1)<\frac{\left(\mathrm{f}\left(\mathrm{r}^{\prime}\right)-\mathrm{f}(\mathrm{r})\right.}{T i}
$$

Si la expresión (10) se cumple, entonces se establece el conjunto $r^{\prime}$ como el nuevo conjunto de rutas que solucionan el problema en estudio. De lo contrario, la solución $r$ permanece como la solución actual. Posteriormente, se procede a evaluar si el número máximo de iteraciones para ese nivel de temperatura se ha alcanzado ( $>$ Nmax). En caso de que la condición se cumpla se evalúa si la temperatura inicial es igual a la temperatura final (Ti=Tf) y este conjunto de rutas $r$ se establece como la solución para el ruteo para la recogida de medicamentos sobrantes y suministro. De lo contrario, se multiplica el factor de enfriamiento $(\alpha)$ utilizando la Eq.(11):

$$
T i=\propto * T i
$$

La nueva temperatura Ti que se calcula permite ejecutar las $\mathrm{n}$ iteraciones del metaheurístico en ese nivel. Una vez se establece la nueva, $\mathrm{Ti}$, se regresa a la etapa que consiste en generar un conjunto de rutas implementando movimientos de intercambio e inserción sobre las rutas $r$ actual. Subsiguientemente, se ejecutan las demás etapas del metaheurístico de enfriamiento simulado antes descritas.

\section{Estudio experimental}

Un estudio experimental es planeado y ejecutado para analizar cuáles factores del problema de ruteo para la recogida de medicamentos sobrantes y suministros tienen un efecto significativo sobre tiempo de la operación (variable respuesta). Adicionalmente, el estudio experimental permite evaluar el desempeño de los metaheurísticos de enfriamiento simulado y enjambre de partículas (PSO) en diferentes condiciones operacionales para el problema de ruteo en análisis. Los factores que son analizados son: estrategia de ruteo (enfriamiento simulado o enjambre de partículas PSO), tamaño de flota de carritos de recogida de medicamentos), tamaño de la lista de los medicamentos sobrantes o suministros a recoger desde las habitaciones (F3) así como número de habitación en el hospital en estudio (F4).

Para realizar el estudio un diseño de experimentos factorial completo con cuatro factores. Este diseño es seleccionado porque no existen restricciones a la aleatorización en el problema de ruteo para la recogida de medicamentos sobrantes y suministros. A continuación, en la Tabla 5, se representa la estructura del diseño de experimentos: De la planeación del diseño experimental se establece que 48 corridas serán ejecutadas en el modelo computacional que integra los metaheurísticos de enjambre de partículas y el PSO además los otros tres factores seleccionados para el problema de ruteo en estudio. La unidad experimental contiene las características del hospital que se detalla en la sección descripción del problema.

Tabla 5: Diseño del estudio experimental

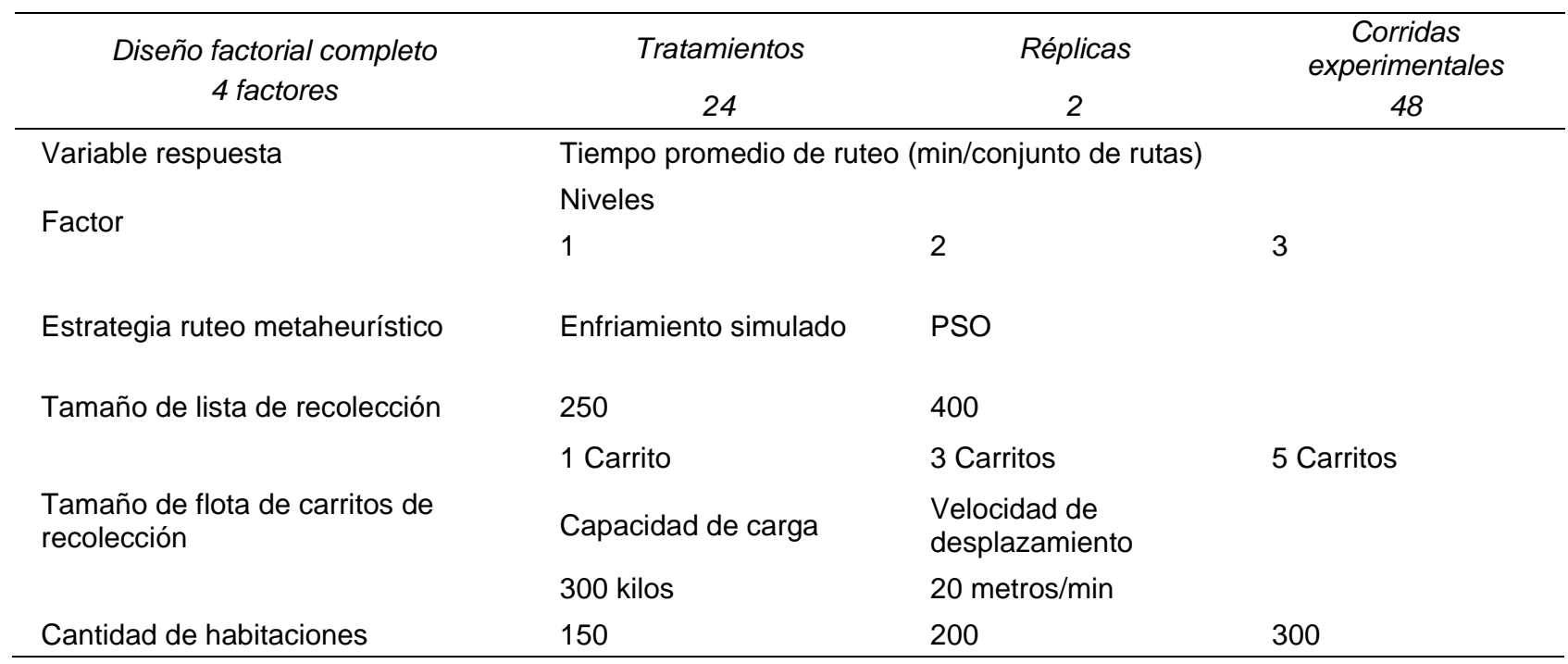


Los parámetros del metaheurístico PSO son i) 1000 iteraciones, componente cognitivo $\left(\mathrm{c}_{1}\right)=0.5$, ii) componente social $\left(\mathrm{c}_{2}\right)=2$ y iii) factor de inercia $\omega=0.7$. Por su parte los parámetros para el metaheurístico de enfriamiento simulado son i) 1000 iteraciones, ii) temperatura inicial es aleatoriamente generada y iii) factor de enfriamiento a igual a 0.90 . Los parámetros elegidos fueron seleccionados a partir de un estudio experimental con 1500 corridas, en el cual, se evaluó y determinó que generaban el mejor desempeño para solucionar el problema en estudio. Por último, el modelo computacional para el problema de ruteo incluyendo los metaheurísticos fueron programados en Java usando NetBeans IDE 8.1. En tanto, los experimentos fueron ejecutados en un computador portátil Intel Quad Core $2.33 \mathrm{GHz}$ procesador y 8GB RAM.

\section{ANÁLISIS Y DISCUSIÓN}

Una vez son ejecutadas las 48 corridas en el modelo para resolver el problema de ruteo para la recogida de los medicamentos sobrantes y los suministros, se calcula la tabla de análisis de varianza (ANOVA) utilizando el diseño de experimentos factorial completo planeado en la Tabla 6:

Tabla 6: ANOVA problema de ruteo en estudio, Minitab 17®

\begin{tabular}{lrrrrr}
\hline Fuente & G.L & \multicolumn{1}{c}{ SS } & \multicolumn{1}{c}{ MS } & Valor $F$ & Valor $P$ \\
\hline Model & 14 & 55484,5 & 3963,2 & 4805,91 & 0,000 \\
ERM & 1 & 2,6 & 2,6 & 3,12 & 0,086 \\
TLR & 1 & 22056,6 & 22056,6 & 26746,75 & 0,000 \\
TFCR & 2 & 27743,0 & 13871,5 & 16821,15 & 0,000 \\
CH & 1 & 4950,0 & 4950,0 & 6002,52 & 0,000 \\
ERM $^{*}$ TLR & 1 & 25,1 & 25,1 & 30,45 & 0,000 \\
ERM $^{*}$ TFCR & 2 & 3,1 & 1,6 & 1,90 & 0,165 \\
ERM $^{*}$ CH & 1 & 0,2 & 0,2 & 0,20 & 0,657 \\
TLR $^{*}$ TFCR & 2 & 569,6 & 284,8 & 345,38 & 0,000 \\
TLR $^{*} \mathrm{CH}$ & 1 & 7,5 & 7,5 & 9,12 & 0,005 \\
TFCR $^{*} \mathrm{CH}$ & 2 & 126,8 & 63,4 & 76,88 & 0,000 \\
Error & 33 & 27,2 & 0,8 & & \\
\hline
\end{tabular}

De la ANOVA calculada en la Tabla 6 , se detecta que factores de tamaño de lista de recogida (TLR), tamaño de flota de carritos de recogida (TFCR) y cantidad de habitaciones en el hospital tienen un efecto significativo sobre el tiempo de ruteo para la recogida de los suministros médicos ya que su valor $p$ es menor a 0.05 (criterio de rechazo). Adicionalmente, las interacciones dobles, entre la estrategia de ruteo con metaheurísticos (ERM) con el factor TLR, TLR con TFCR, TLR con $\mathrm{CH}$ y TFCR ${ }^{*} \mathrm{CH}$ también tienen un efecto significativo sobre el tiempo de ruteo en el problema en estudio debido a que su valor $p$ es menor a 0.05 . Por los motivos expuestos para estudiar el efecto de los factores del problema de ruteo se analizan las interacciones dobles detectadas como significativas.

\section{EMR*TLR $^{*}$}

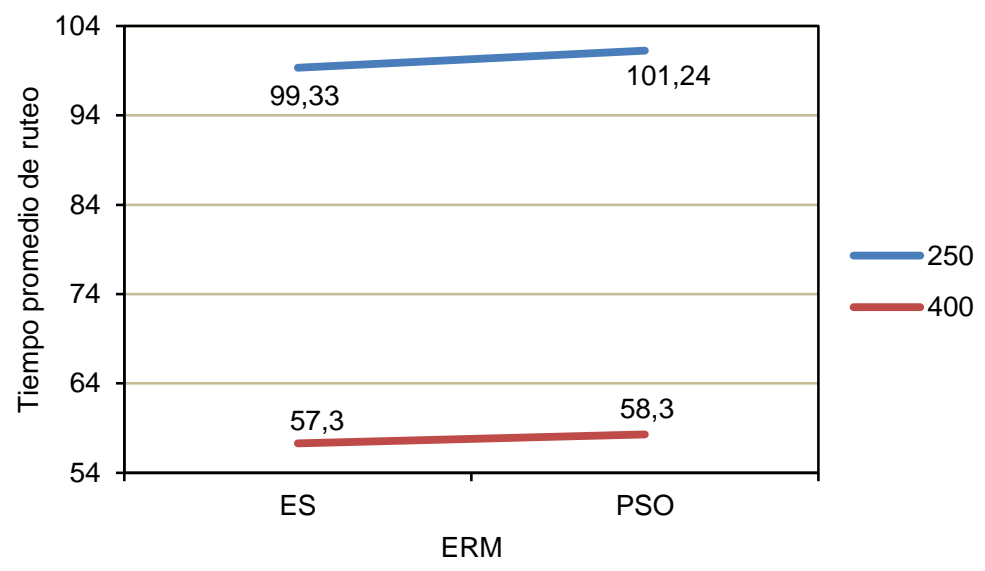

Fig. 4: Interacción doble entre ERM y TLR, Minitab 17® 
De la interacción entre los factores estrategia de ruteo con metaheurísticos (ERM) con el factor TLR de la figura 4, se detecta que las combinaciones de los niveles metaheurístico PSO con el nivel TLR de 250 medicamentos sobrantes o suministros genera menores tiempos de recogida que la combinación del nivel metaheurístico RS con valores de 58,30 y 57,30 minutos / conjunto de ruta. Estas diferencias generan un ahorro de $1 \mathrm{~min} /$ conjunto de rutas de recogida. De otra parte, para un nivel del factor TLR de 400 medicamentos sobrantes o suministros a recoger la combinación con el nivel metaheurístico RS produce el menor tiempo promedio de ruteo que la combinación con el nivel metaheurístico PSO con valores de 99,33 y 101,24 minutos /conjunto de rutas. Estas diferencias entre los niveles de factores producen un ahorro de aproximadamente 2 minutos para conjunto de rutas ejecutadas en el hospital. A partir de estos resultados se puede inferir que el metaheurístico PSO genera un mejor desempeño para TLR de menor tamaño que el metaheurístico RS y viceversa para el ruteo para la recogida de medicamentos sobrantes y suministro de menor tiempo posible. Por lo tanto, debe considerarse estos resultados estadísticos para la toma de decisiones para este problema de ruteo en hospitales independiente de la cantidad de habitaciones $(\mathrm{CH})$, ya que la interacción con el ERM no fue significativa estadísticamente.

A partir del análisis estadístico de las interacciones dobles entre TLR y TFCR de la figura 5 , se detecta que la combinación del nivel 5 carros recolectores con el nivel TLR de 250 medicamentos sobrantes o suministros reduce el tiempo promedio de ruteo en $44 \%$ ( 26,25 minutos/conjunto de rutas) y $61 \%$ (50,04 minutos/conjunto de rutas) respecto a las combinaciones de niveles que emplean 3 y 1 carro recolector para ejecutar la operación en el hospital. Con respecto a las combinaciones de 5 carros recolectores con una TLR de 400 medicamentos sobrantes se identifica que esta disminuye el tiempo promedio de ruteo en 34 (35,29 minutos) y $51 \%(68,15$ minutos) con relación a tamaños de flota con 1 y 3 carros recolectores respectivamente. Estos resultados apoyan decisiones de diseño de ruteo en el hospital para ejecutar las actividades según los tiempos de recogida planeados o establecer los menores costos logísticos ya que a menor cantidad de TFCR se espera un menor costo total, pero tiempos más largos de ruteo.

Del análisis de la interacción doble entre TFCR y $\mathrm{CH}$, se identifica que las combinaciones del nivel 5 carros recolectores en un hospital con 150 habitaciones, se disminuye el tiempo promedio de conjunto de rutas en $32,26(0-45 \%)$ y 57,19 minutos (o $-59 \%$ ) con relación a los niveles 1 y 3 carros recolectores respectivamente. Adicionalmente, las combinaciones del nivel 5 carros recolectores en un hospital con 300 habitaciones se disminuye el tiempo promedio de conjunto de rutas en $29,29(-33 \%)$ y 61,38 minutos $(-51 \%)$ con respecto a los niveles 1 y 3 carros recolectores. Estos resultados estadísticos sirven de base para tomar decisiones de capacidad y costos logísticos para solucionar el problema de ruteo para la recogida de medicamentos sobrantes y suministros según el tamaño del hospital y tamaño de la flota de recolectores.

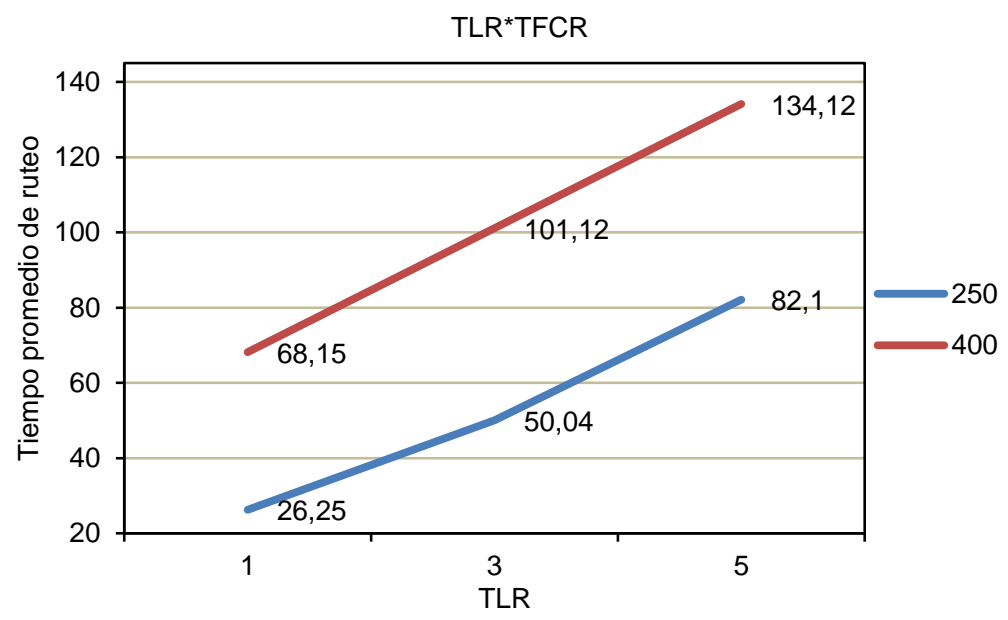

Fig. 5: Interacción doble entre TFCR y TLR, Minitab 17®

\section{DISCUSIÓN FINAL}

En logística hospitalaria, la formulación y solución de problemas de ruteo para la recogida de medicamentos y suministros sobrantes, de los servicios de las habitaciones de mínimo tiempo posible, contribuyen al incremento de la productividad como fue demostrado estadísticamente. Desde la perspectiva científica el problema de ruteo desarrollado en logística hospitalaria es un enfoque novedoso en la literatura ya que no se detectó en los antecedentes, metaheurísticos o modelos de optimización que aborden el problema en estudio en la logística hospitalaria para resolver un problema que considere $n$ medicamentos o suministros sobrantes 
i y una flota de $K$ carros recolectores homogéneos (igual capacidad de carga y características técnicas). Adicionalmente, en el modelamiento se incluyen factores como diferentes tamaños de listas de recolección (250 y 400 productos) así como hospitales con diferentes cantidades de habitaciones (150 y 300).

De la validación experimental usando un diseño factorial completo de cuatro factores se detectó estadísticamente el factor estrategia con ruteo con metaheurísticos tiene un efecto significativo sobre el tiempo promedio para la recogida de los medicamentos sobrantes y los suministros. Del análisis estadístico se identificó que las combinaciones de los niveles metaheurístico PSO con el nivel TLR de 250 medicamentos sobrantes o suministros genera menores tiempos de recogida que la combinación del nivel metaheurístico RS con valores de 58,30 y 57,30 minutos / conjunto de ruta lo que produce un ahorro de 1 min/conjunto. De otra parte, para un TLR de 400 medicamentos o suministros a recolectar la combinación con el nivel metaheurístico RS produce el menor tiempo promedio de ruteo que la combinación con el nivel metaheurístico PSO con valores de 99,33 y 101,24 minutos /conjunto de rutas generando un ahorro de aproximadamente 2 minutos.

Desde la perspectiva operacional implica que los niveles de los factores de estas interacciones deben ser configurados según la cantidad de habitaciones del hospital, el tamaño de la lista de recolección y la estrategia de ruteo con metaheurísticos con el fin de obtener el menor tiempo posible lo cual impacta en la efectividad de la logística hospitalaria. Las mejores soluciones obtenidas de la experimentación fueron las combinaciones de niveles de 5 carros recolectores con el nivel TLR de 250 medicamentos sobrantes o suministros disminuye el tiempo promedio de ruteo en $44 \%$ (26,25 minutos/conjunto de rutas) y $61 \%(50,04$ minutos/conjunto de rutas) respecto a los otros dos niveles de TFCR. Con relación a la combinación de 5 carros recolectores con una TLR de 400 medicamentos sobrantes se identifica estas reduce el tiempo promedio de ruteo en 34 (35,29 minutos) y $51 \%(68,15$ minutos) respecto a los otros dos tamaños de flota modelados.

\section{CONCLUSIONES}

De acuerdo a los resultados obtenidos, se plantean las siguientes conclusiones principales:

Para resolver el problema de ruteo para la recogida de medicamentos y suministros sobrantes de los servicios de las habitaciones de mínimo tiempo posible se desarrollaron y validaron dos metaheurísticos denominados enfriamiento simulado (ES) y optimización de enjambre de partículas (OEM). Los metaheurísticos permiten solucionar el problema para hospitales con más de 150 habitaciones los cuales pueden ser clasificados como medianos. Debe resaltarse que en la literatura científica no se identificaron artículos científicos que desarrollen metaheurísticos o modelos de optimización para abordar el problema en estudio en la logística hospitalaria, de allí el aporte alcanzado con el presente artículo.

De la validación experimental usando un diseño factorial completo de cuatro factores se detectó estadísticamente el factor estrategia con ruteo con metaheurísticos tiene un efecto significativo sobre el tiempo promedio de recogida de los medicamentos sobrantes y los suministros. Del análisis estadístico se identificó que las combinaciones de los niveles metaheurístico PSO con el nivel TLR de 250 medicamentos sobrantes o suministros genera menores tiempos de recogida que la combinación del nivel metaheurístico ES con valores de 58,30 y 57,30 minutos / conjunto de ruta lo que produce un ahorro de $1 \mathrm{~min} /$ conjunto de rutas. De otra parte, para un TLR de 400 medicamentos o suministros a recolectar la combinación con el nivel metaheurístico ES produce el menor tiempo promedio de ruteo que la combinación con el nivel metaheurístico PSO con valores de 99,33 y 101,24 minutos /conjunto de rutas generando un ahorro de aproximadamente 2 minutos.

Del análisis estadístico, también se identificó que las interacciones dobles TLR (tamaño de lista de recolección) y TFCR (Tamaño de Flota de Carros recolectores) tienen un efecto significativo sobre el tiempo promedio del conjunto de rutas de recogida de los medicamentos sobrantes y suministros. Desde la perspectiva operacional implica que los niveles de los factores de estas interacciones deben ser configurados según la cantidad de habitaciones del hospital, el tamaño de la lista de recolección y la estrategia de ruteo con metaheurísticos con el fin de obtener el menor tiempo posible lo cual impacta en la efectividad de la logística hospitalaria. Las mejores soluciones obtenidas de la experimentación fueron las combinaciones de niveles de 5 carros recolectores con el nivel TLR de 250 medicamentos sobrantes o suministros disminuye el tiempo promedio de ruteo en $44 \%$ (26,25 minutos/conjunto de rutas) y $61 \%(50,04$ minutos/conjunto de rutas) respecto a los otros dos niveles de TFCR. Con relación a la combinación de 5 carros recolectores con una TLR de 400 medicamentos sobrantes se identifica estas reduce el tiempo promedio de ruteo en 34 (35,29 minutos) y $51 \%(68,15$ minutos $)$ respecto a los otros dos tamaños de flota modelados.

Finalmente, como trabajos futuros se sugiere la inclusión de otros factores como ubicación del almacén central en el hospital y nivel de competencia de los operadores de la recolección, así como desarrollar otros metaheurísticos que permitan evaluar el desempeño del ES y el PSO buscando obtener menores tiempos para el ruteo para la recogida de los medicamentos sobrantes y suministros en logística hospitalaria. 


\section{AGRADECIMIENTOS}

Los autores del presente trabajo agradecen a la Pontificia Universidad Javeriana de Bogotá, por el apoyo para la realización del proyecto de investigación y asignación de recursos para los productos de investigación.

\section{REFERENCIAS}

Al-Qatawneh, L. y K. Hafeez, Healthcare logistics cost optimization using a multi-criteria inventory classification, Proceedings of the 2011, International Conference on Industrial Engineering and operations management, Kuala Lumpur (2011)

Battini, D., E. Hassini y otros cinco autores, Drug inventory management and distribution: outsourcing logistics to thirdparty providers, Strategic Outsourcing: An International Journal, 6 (1), 48-64 (2013)

Bhakoo, V., P. Singh y A. Sohal, Collaborative management of inventory in Australian hospital supply chains: practices and issues, Supply Chain Management: An International Journal, 17 (2), 217-230 (2012)

Ganesh, K., T. T. Narendran y S. P. Anbuudayasankar, Evolving cost-effective routing of vehicles for blood bank logistics, International Journal of Logistics Systems and Management, 17 (4), 381-415 (2014)

Jlassi, J., J. Euchi y H. Chabchoub, Dial a ride and emergency transportation problems in ambulance services, Computer Science and Engineering, 2 (3), 17-23 (2012)

Kergosien, Y., C. Lenté, J.-C. Billaut y S. Perrin, Metaheuristic algorithms for solving two interconnected vehicle routing problems in a hospital complex, Computers and Operations Research, 40 (10) 2508-2518 (2013)

Kriegel, J., F. Jehle, M. Dieck y P. Mallory, Advanced services in hospital logistics in the German health service sector, Logistics Research, 6(2-3), 47-56 (2013)

Park, JS., HS. Kim y otros tres autores, A Study on the Development of Integrated Logistics System for Hospital Logistics Cooperation, Journal of the Korea Safety Management and Science, 16(2), 139-46 (2014)

Rodríguez, A., L. Ramirez y F. Basile, Solución Metaheurística para el Problema de Enrutamiento (RWA) en Redes de Ópticas de Multiplexación por División de Longitud de Onda (WDM), doi: 10.4067/S0718-07642017000600015, Información Tecnológica, 28(6), 133-146 (2017)

Suarez, V., W. Sarache e Y. Costa, Una Solución al Enrutamiento de Vehículos en Ciudades Montañosas Considerando Aspectos Ambientales y Económicos, doi: 10.4067/S0718-07642018000300003, Información Tecnológica, 29(3), 3-14 (2018)

Supakdee, K., N. Nanthasamroeng y R. Pitakaso, Clustering Locating Routing Algorithm for Vehicle Routing Problem: An Application in Medical Equipment Maintenance, Proceedings of the Institute of Industrial Engineers Asian Conference, 965-973 (2013)

Talbi, E., Metaheuristics from design to implementation, Wile, New Jersey (2009) 